\title{
Estado nutricional en pacientes críticos como predictor de morbimortalidad en un Hospital Nacional de EsSalud de Lima-Perú
}

\author{
Nutritional status in critical patients as a predictor of morbidity and mortality in a National Hospital \\ of Essalud in Lima-Peru
}

Jossiel Roque Aycachi', María Miranda Flores²

\begin{abstract}
RESUMEN
Objetivo: El objetivo del trabajo fue determinar la asociación de la morbimortalidad con el estado nutricional de pacientes que ingresan a la Unidad de Cuidados Especiales (UCE) de Emergencia del Hospital Nacional Guillermo Almenara Irigoyen. Material y métodos: El estudio es de diseño no experimental, de corte longitudinal, de alcance observacional analítico de cohortes retrospectivos. El muestreo fue de tipo no probabilístico, por conveniencia. El grupo se conformó por 124 pacientes, quienes fueron divididos en dos grupos: desnutridos $(\mathrm{n}=31)$ y normonutridos $(\mathrm{n}=93)$ para la observación de ambos grupos, frente a la exposición (ventilación mecánica, infección, estancia hospitalaria, estado de egreso). Resultados: Se encontró una relación significativa y un odds ratio entre el estado nutricional y la ventilación mecánica $(\mathrm{OR}=2.6 ; \mathrm{p}<0.033)$, infecciones $(\mathrm{OR}=2.6$; $\mathrm{p}<0.038)$, estancia hospitalaria $(\mathrm{OR}=4.154 ; \mathrm{p}<0.01)$ y estado de egreso $(\mathrm{OR}=3.646 ; \mathrm{p}<0.004)$. Conclusiones: Finalmente, se llega a la conclusión de la existe una relación significativa entre las variables estudiadas.
\end{abstract}

Palabras clave: Estado nutricional; desnutrición; morbimortalidad.

\begin{abstract}
Objetive: The objective was to determine the association of morbidity and mortality in the nutritional status of patients admitted to the Special Care Unit (SCU) Emergency of Hospital Nacional Guillermo Almenara Irigoyen. Methods: The study is not experimental design, slitting, powerful analytical observational retrospective cohort. The sampling was non probabilistic for convenience. The group was formed by 124 patients, who were divided into two groups, malnourished $(\mathrm{n}=31)$ and normally nourished $(\mathrm{n}=93)$ for the observation of both groups from exposure (mechanical ventilation, infection, hospital stay, discharge status). Results: Was found a significant relationship and an odds ratio between nutritional status and mechanical ventilation $(\mathrm{OR}=2.6 ; \mathrm{p}<0.033)$, infections $(\mathrm{OR}=2.6 ; \mathrm{p}<0.038)$, hospital stay $(\mathrm{OR}=4.154 ; \mathrm{p}<0.01)$ and discharge status $(\mathrm{OR}=3.646 ; \mathrm{p}<0.004)$. Conclusions: Finally, we reach the conclusion of a significant relationship between the studied variables.
\end{abstract}

Keywords: Nutritional status, malnutrition, morbidity and mortality.

\footnotetext{
${ }^{1}$ Licenciado en Nutrición Humana. Universidad Peruana Unión. Lima, Perú.

${ }^{2}$ Magíster en Nutrición y Bioquímica. Directora académica de la EP de Nutrición Humana. Universidad Peruana Unión. Lima Perú.
} 


\section{INTRODUCCIÓN}

En la actualidad, la prevalencia de desnutrición, a nivel mundial en hospitales, oscila entre un 10 a $80 \%$. Haciendo, de esta condición, una determinante que interfiere con el proceso de recuperación, favoreciendo un desenlace fatal. Estos indicadores no han cambiado a lo largo del tiempo, por tanto, para ninguno es sorpresa que los pacientes quienes permanecen en el hospital, empeoran su estado nutricional. Esta descripción se corrobora con la descrita por Naber (1997), donde evaluó a 155 pacientes hospitalizados, y llegó a la conclusión de que la aparición de complicaciones, durante la estancia hospitalaria, se relaciona con la gravedad de la desnutrición.

En Estados Unidos, la prevalencia de desnutrición varía entre 25 y $60 \%$ (Kelly, 2000), y se evidencia por la pérdida de peso durante su hospitalización. En Sudamérica, en países como Brasil, Waitzberg (2001), en un estudio multicéntrico con 4000 pacientes, reportaron cifras de desnutrición de 48.1\%, y en Argentina, Wyszynski (2003) encontró cifras del $47 \%$. En el Perú, las cifras son próximas e incluso mayores, según Ortiz (2007) quien reportó una prevalencia de desnutrición del $52.8 \%$.

La evaluación del estado nutricional, de los pacientes hospitalizados, viene siendo considerada como parte de la intervención nutricional. Sin embargo, en los hospitales existe un grupo reducido que necesita de cuidados intensivos, y una intervención oportuna. El estado nutricional, en estos pacientes críticos, es fundamental, debido a la respuesta de mecanismos metabólicos y catabólicos que condicionan su pronóstico, y más aún, si presenta algún grado de desnutrición. Por tanto, la desnutrición, en este tipo de pacientes, puede considerase como un factor predisponente de morbimortalidad. En otros servicios, se ha observado que una buena intervención nutricional mejora la condición y respuesta frente a la agresión y por ende, un mejor pronóstico. Por tanto, todo paciente admitido en un hospital tiene el derecho a esperar que sus requerimientos nutricionales sean provistos. Debido a que se conoce que, si más temprano se aplica el soporte nutricional, mejor será la respuesta evolutiva del paciente. La mayoría de pacientes críticos, si fueran sometidos a una intervención nutricional, de manera oportuna durante su período de estancia en la unidad de cuidados intensivos, beneficiarían su recuperación. Por muchos años se ha venido concientizando, a los profesionales de salud, sobre la prevalencia de la desnutrición hospitalaria y sus consecuencias en la morbimortalidad, la estancia hospitalaria, el riesgo de infecciones y las complicaciones en general.
Por tanto, el objetivo de estudio es determinar la asociación de la morbimortalidad con el estado nutricional de pacientes que ingresan a la Unidad de Cuidados Especiales (UCE) de Emergencia del Hospital Nacional Guillermo Almenara Irigoyen, a fin de establecer si la exposición a tal factor representa un mayor riesgo para la aparición del evento.

\section{MATERIAL Y MÉTODOS}

El presente trabajo de investigación es de diseño no experimental, de corte longitudinal, de alcance observacional analítico de cohortes retrospectivos. La población estuvo conformada por 598 pacientes que ingresaron a la Unidad de Cuidados Especiales (UCE) del Hospital Guillermo Almenara Irigoyen, en los meses de enero a julio del 2014, con una estadía mayor a 48 horas, y que su estado clínico permitiera realizar las mediciones para la evaluación del estado nutricional. La muestra está conformada por 158 pacientes, seleccionados por conveniencia y de acuerdo a los criterios exclusión, quedando 124 pacientes para el estudio. De los cuales el 51,6\% de pacientes era de género masculino y $48,4 \%$ de género femenino.

En cuanto a las variables dependientes de morbimortalidad, el 78,2\% (97 pacientes) recibió ventilación mecánica, 83,1\% (103 pacientes) presentó al menos un cuadro de infección, la estancia hospitalaria promedio fue de 11,4 días (DS 4,2), presentándose en el 71,8\% (89 pacientes) una estancia mayor de 12 días. La mortalidad de los pacientes se presentó en el 48,4\% (60 pacientes) de la muestra estudiada.

\section{Técnicas e instrumentos de recolección de datos}

Los datos se obtuvieron de la historia clínica nutricional, que consta de 5 ítems incluyendo (1) datos generales y morbilidades, (2) evaluación nutricional, (3) plan de alimentación, (4) valoración global subjetiva y (5) el seguimiento nutricional. Se tomaron las medidas antropométricas (índice de masa corporal), bioquímicas (albúmina) y evaluación clínica (prueba de valoración global subjetiva) para la evaluación del estado nutricional. Estas medidas fueron realizadas de manera integral formando dos grupos:

Normonutrido: Pacientes con valoración nutricional adecuada, según los datos antropométricos (índice de masa corporal, valoración global subjetiva), clínicos (valoración global subjetiva) y bioquímicos (albúmina). 
Desnutridos: Pacientes con valor nutricional menores a los valores normales.

Asimismo se consideró, según el diseño del estudio, los siguientes factores de exposición: Ventilación mecánica (como la necesidad de uso de ventilación mecánica), infecciones (cultivo positivo en la estancia, sin la evidencia que la infección estuviese presente al momento de ingreso), estancia hospitalaria (número de días de permanencia en la UCE) y el estado de egreso (como vivo o fallecido).
El proceso de recolección de datos, así como el procesamiento y análisis estadístico, fue ejecutado por el investigador, haciendo uso del paquete estadístico SPSS versión 21.0, considerándose el análisis descriptivo da cada una de las variables de estudio, y análisis bivariado para observar frecuencias y posibles asociaciones significativas, utilizando la prueba de chi-cuadrado de Pearson con cálculo de odds ratio e intervalo de confianza al $95 \%$.

\section{RESULTADOS}

\section{Tabla 1}

Evaluación nutricional y ventilación mecánica

\begin{tabular}{lllllll}
\hline Estado nutricional & \multicolumn{2}{l}{ Ventilación mecánica } & \multicolumn{3}{l}{ Total } \\
\cline { 2 - 6 } & Sí & & No & & \\
& $\mathrm{N}$ & $\%$ & $\mathrm{~N}$ & $\%$ & $\mathrm{~N}$ & $\%$ \\
\hline Desnutridos & 77 & 79,4 & 16 & 59,3 & 93 & 75 \\
\hline Normonutridos & 20 & 20,6 & 11 & 40,7 & 31 & 25 \\
\hline Total & 97 & 100 & 27 & 100 & 124 & 100 \\
\hline
\end{tabular}

\section{Tabla 2}

Odds ratio y significancia de la ventilación mecánica en el estado nutricional.

\begin{tabular}{llll}
\hline Variable & OR & IC 95\% & Valor $p$ \\
\hline Ventilación mecánica & 2,647 & $(1,064-6,587)$ & $<0,033$ \\
\hline
\end{tabular}

En la tabla 1 y 2 se muestra que de los 124 pacientes, considerando la necesidad de ventilación mecánica, los pacientes normonutridos necesitaron de la ventilación mecánica en un 20,6\%, mientras que los pacientes desnutridos necesitaron en un $79,4 \%$. Teniendo un incremento de 2,6 veces de presentarse la necesidad de la ventilación mecánica en pacientes desnutridos, en comparación con los normonutridos. Por lo tanto, existe asociación significativa entre el estado nutricional y la ventilación mecánica, con un valor $\mathrm{p}<0,033$.

Tabla 3

Evaluación nutricional e infecciones

\begin{tabular}{lllllll}
\hline Estado nutricional & \multicolumn{2}{l}{ Infecciones } & \multicolumn{3}{l}{ Total } \\
\cline { 2 - 5 } & Sí & & No & & \\
& N & $\%$ & N & $\%$ & N & $\%$ \\
\hline Desnutridos & 81 & 78,6 & 12 & 57,1 & 93 & 75 \\
\hline Normonutridos & 22 & 21,4 & 9 & 42,9 & 31 & 25 \\
\hline Total & 97 & 100 & 27 & 100 & 124 & 100 \\
\hline
\end{tabular}


Tabla 4

Odds ratio y significancia de infecciones en el estado nutricional.

\begin{tabular}{llll}
\hline Variable & OR & IC $95 \%$ & Valor $p$ \\
\hline Infecciones & 2,671 & $(1,032-7,390)$ & $<0,038$ \\
\hline
\end{tabular}

En la tabla 3 y 4 se observa que de los 124 pacientes, considerando la presencia de, al menos, un proceso infeccioso, solo 22 pacientes normonutridos presentaron un proceso infeccioso en un $21,4 \%$, mientras que el $78,6 \%$ pacientes desnutridos presentaron un proceso infeccioso. Teniendo un incremento de 2,6 veces de presentarse algún proceso infeccioso. Encontrándose una asociación significativa entre el estado nutricional y los procesos infecciosos, con un valor $\mathrm{p}<0,038$.

Tabla 5

Estado nutricional y estancia hospitalaria.

\begin{tabular}{lllllll}
\hline Estado nutricional & \multicolumn{3}{l}{ Estancia hospitalaria } & \multicolumn{3}{l}{ Total } \\
\cline { 2 - 6 } & Mayor 12 & \multicolumn{3}{l}{ Menor 12 } & & \\
& $\mathrm{N}$ & $\%$ & $\mathrm{~N}$ & $\%$ & $\mathrm{~N}$ & $\%$ \\
\hline Desnutridos & 74 & 83,1 & 19 & 54,3 & 93 & 75 \\
\hline Normonutridos & 15 & 16,9 & 16 & 45,7 & 31 & 25 \\
\hline Total & 89 & 100 & 35 & 100 & 124 & 100 \\
\hline
\end{tabular}

Tabla 6

Odds ratio y significancia de la estancia hospitalaria en el estado nutricional.

\begin{tabular}{llll}
\hline Variable & OR & IC 95\% & Valor $p$ \\
\hline Estancia hospitalaria & 4,154 & $(1,747-9,878)$ & $<0,01$ \\
\hline
\end{tabular}

En la tabla 5 y 6 se muestra que de los 124 pacientes, el $83,1 \%$ (74 pacientes) tuvo una estancia hospitalaria mayor a 12 días, mientras que en los pacientes normonutridos fue del 16,9\% (15 pacientes). Teniendo un incremento de 4,1 veces de presentarse una estancia hospitalaria mayor a 12 días. Por tanto, existe asociación significativa entre el estado nutricional y la estancia hospitalaria, con un valor $\mathrm{p}<0,01$.

Tabla 7

Estado nutricional y estado de egreso

\begin{tabular}{lllllll}
\hline Estado nutricional & \multicolumn{2}{l}{ Estado de egreso } & \multicolumn{3}{l}{ Total } \\
\cline { 2 - 6 } & Fallecido & \multicolumn{3}{l}{ Vivo } & & \\
& $\mathrm{N}$ & $\%$ & $\mathrm{~N}$ & $\%$ & $\mathrm{~N}$ & $\%$ \\
\hline Desnutridos & 52 & 86,7 & 41 & 64,1 & 93 & 75 \\
\hline Normonutridos & 8 & 13,3 & 23 & 35,9 & 31 & 25 \\
\hline Total & 60 & 100 & 64 & 100 & 124 & 100 \\
\hline
\end{tabular}

Tabla 8

Odds ratio y significancia del estado de egreso en el estado nutricional

\begin{tabular}{llll}
\hline Variable & OR & IC 95\% & Valor $p$ \\
\hline Estado de egreso & 3,646 & $(1,479-8,992)$ & $<0,004$ \\
\hline
\end{tabular}

En la tabla 7 y 8 se observa que de los 124 pacientes, el estado de egreso medido, como vivo o fallecido, se observó que en el nivel de mortalidad solo se presentó en 8 pacientes $(13,3 \%)$ del grupo de normonutridos, mientras que en el grupo de desnutridos fue del $86,7 \%$. Teniendo un incremento de 3,6 veces de que los pacientes desnutridos fallezcan. Encontrándose una asociación significativa entre el estado nutricional y el estado de egreso, con un valor $\mathrm{p}<0,004$. 


\section{DISCUSIÓN}

En el estudio se encontró que la mayoría de pacientes críticos presentó algún grado de desnutrición, debido fundamentalmente al estado hipercatabólico e hipermetabólico propio en este tipo de pacientes. Asimismo, en un estudio se evaluó el estado nutricional de pacientes críticos, encontrando que el $84,2 \%$ presentaba algún grado de desnutrición, confirmando las altas prevalencias de desnutrición en estos pacientes (Vásquez, Davas, \& Reyes, 2012). A diferencia de otras unidades, donde se demuestra que, a nivel médico y quirúrgico, la prevalencia de desnutrición fue de 40,2\% (Vidal, Iglesias, Pertega, Ayúcar, \& Vidal, 2008), de igual manera Barreto (2012) reportó una prevalencia de $37,8 \%$. Encontrando diferencias de prevalencias de desnutrición en diversos servicios, siendo alta en pacientes críticos, debido al tipo de población y a los diferentes criterios diagnósticos, pueden existir discrepancias en las prevalencias de desnutrición hospitalaria en diferentes estudios (Santana-Cabrera, Shanahan, García, Ramirez, Sánchez, \& Hernández, 2006), justificándose la demanda del servicio en atención de pacientes con patologías crónicas y estados críticos.

El estudio también mostró una asociación significativa entre el estado nutricional y la ventilación mecánica $(\mathrm{OR}=2,6 ; \mathrm{p}<0,033)$. Estos resultados son corroborados por investigadores, como Hernández (2011), quien estudió la asociación entre la desnutrición y la ventilación mecánica, llegando a la conclusión de que los pacientes con desnutrición tuvieron un tiempo mayor de uso de ventilación mecánica $(\mathrm{p}=0,037)$. Asimismo, Frutos (2003), en su estudio cuyo objetivo era conocer las variables asociadas a la mortalidad de los pacientes que precisan de ventilación mecánica, comprobó que la duración del tiempo del uso de ventilación mecánica, influía en la mortalidad de los pacientes.

Por lo tanto, se cataloga a la desnutrición como un problema grave en pacientes críticos, porque estos requieren de mayor apoyo ventilatorio (Duarte, León, Larrondo, Crespo, Segura, \& Ernesto, 2010). Además de la desnutrición, el aporte calórico del paciente crítico es menor que el recomendable, llegando a un balance calórico negativo. Un estudio midió el gasto energético mediante calorimetría indirecta en estos pacientes, realimentándolos con un aumento del balance energético, acompañándose de ganancia de peso (Ryan, Road, Buckey, Ross, \& Whittaker, 1993). Sin embargo, más que lograr la ganancia de peso, Rogers (1992) evaluó el efecto fisiológico de la alimentación, aportando las calorías y proteínas ajustadas a las necesidades metabólicas, mejorando la función muscular respiratoria, aunque aún no está establecido que mejore los parámetros respiratorios o la supervivencia, encontrando un grado de asociación, mas no una relación directa.

Asimismo, se encontró una asociación significativa entre el estado nutricional y las infecciones, presentándose mayormente en pacientes desnutridos $(\mathrm{OR}=2,6 ; \mathrm{p}<0,038)$. El balance calórico negativo es evidente en este tipo de pacientes, así como lo demuestra Villet (2005), cuyo objetivo de estudio fue evaluar la relación entre el balance energético y las complicaciones en pacientes críticos, encontrándose una mayor incidencia de infecciones en relación con el balance calórico negativo $(\mathrm{p}=0.001)$. Por el contrario, Mault (2000) encontró mejores resultados con un balance calórico positivo. Asimismo, diversos autores concluyen que la desnutrición constituye un factor predisponente para adquirir infección nosocomial, tales como neumonía, infección del tracto urinario, bacteriemia e infecciones de heridas quirúrgicas (Pingleton, 1990) (Christman \& McCain, 1993). Las infecciones nosocomiales son un importante problema de salud pública, incrementando la morbimortalidad en pacientes críticos. Si bien, al no valorar en este trabajo los costes de medicamentos, especialmente antibióticos, usados por pacientes para el tratamiento de procesos infecciosos, en un estudio realizado por Naber (1997) encontró que los pacientes desnutridos consumieron un promedio de 7,7 veces medicamentos/día, frente a 5,1 del grupo de normonutridos. Corroborando que el estado inmunológico del paciente crítico está estrechamente relacionado con el estado nutricional, alterando su estructura, organización y funcionamiento en pacientes desnutridos (Marik \& Flemmer, 2012).

De igual forma, se encontró una asociación significativa entre el estado nutricional y la estancia hospitalaria $(\mathrm{OR}=4,154 ; \mathrm{p}<0,01)$. La media de la estancia fue de 11,4 días. Sin embargo, aún no está definida la estancia prolongada de forma uniforme, hay autores que la definen como una estancia mayor de 10 días (Bashour, Yared, Ryan, Rady, Masha, \& Leventhal, 2000) (Heyland, Konopad, Noseworthy, Johnston, \& Gafni, 1998), y otros que consideran estancias mayores de 28 días (Hughes, MacKirdy, Norrie, \& Grant, 2001) (Montuclard, GarrousteOrgeas, Timsit, Misset, De jonghe, \& Carlet, 2000). En base a la media encontrada, se tomó como corte de estancia hospitalaria, menor y mayor a 12 días, reflejando una diferencia en los días de estancia entre los grupos estudiados. El grupo de desnutridos presentó una estancia mayor a 12 días, en comparación al grupo de normonutridos $(83,1 \%$ vs $16,9 \%$ ). Autores como Barreto (2005), encontró estancias hospitalarias que oscilan entre 6,9 días (DS 
7,3) para pacientes desnutridos, en comparación con los pacientes normonutridos que fue de 4,6 días (DS 5,6). Otro autor, Norman (2008) encontró estancias aún mayores, de 17,2 días para pacientes desnutridos comparado con 9,1 días en pacientes normonutridos. Esto se debe a factores propios de la enfermedad, sumado a que un paciente puede tener más de una morbilidad (ventilación mecánica, infecciones) que pueden influir en la prolongación de su estancia.

Por otro lado, se encontró una asociación significativa entre el estado nutricional y la mortalidad a razón del estado de egreso $(\mathrm{OR}=3,646 ; \mathrm{p}<0,004)$. Debido al contexto del desarrollo de la desnutrición, un empeoramiento de este estado durante la estancia hospitalaria, se ha considerado como un factor predisponente de la mortalidad.

Así como lo demuestra Sullivan (1999), cuyo estudio determinó la correlación entre la ingesta de nutrientes y la mortalidad, encontrando que ingestas menores a los requerimientos de cada pacientes contribuye a un mayor riesgo de mortalidad. De igual forma, Villalobos (2006) observó que la desnutrición es uno de los factores que favorecen el incremento de mortalidad. Explicándose por la pérdida de peso, expresado en una reducción de masa grasa y magra (reservas), atenuando la respuesta del organismo frente a la agresión, pudiendo llegar a muerte. En el presente estudio, la estancia fue mayores a 12 días, pudiendo incrementarse el riesgo en estancias hospitalarias largas. Por tanto, el riesgo de morir, en el presente estudio, de un paciente desnutrido es 3,6 veces mayor, en relación con un paciente normonutrido.
Se concluye que existe una relación significativa entre el estado nutricional y la ventilación mecánica, infecciones, estancia hospitalaria y estado de egreso en pacientes críticos. Observando que los pacientes desnutridos tienen un riesgo de presentar morbimortalidad y demostrando la importancia de la evaluación del estado nutricional en pacientes, con la finalidad de tener una mejor intervención. Asimismo, se encontró una alta prevalencia de desnutrición en la Unidad de Cuidad Especiales (UCE), corroborado con la teoría científica, donde la desnutrición es considerada uno de los principales problemas de salud en todo el mundo, afectando, de manera especial, a pacientes hospitalizados.

Como es de importancia precisar las asociaciones definidas en la presente investigación, se recomienda realizar estudios epidemiológicos de carácter multicéntricos, con una mayor muestra poblacional, de tipo prospectivo para una mejor validez, para conocer el comportamiento de la tendencia de riesgo de estas variables en el tiempo. Se recomienda enfocar la importancia de realizar la evaluación nutricional oportunamente para lograr una intervención eficaz.

Declaración de financiamiento y de conflicto de intereses:

El estudio fue financiado por los autores, quienes declaran no tener algún tipo de conflicto de interés en la investigación realizada.

\section{Correspondencia:}

\section{Jossiel Roque Aycachi}

Universidad Peruana Unión. Carretera Central Km 19.5 Ñaña. Lima. Perú.

e-mail: jossielbriams@gmail.com 


\section{REFERENCIAS BIBLIOGRÁFICAS}

Barreto, J. (2005). State of malnutrition in Cuban hospitals. Nutrition, 21, 487-97.

Barreto, J., Santana, S., Barcelo, M., \& et al. (2012). Estado de la desnutrición en el Hospital Clínico Quirúrgico "Hermanos Ameijeiras". Revista Cubana de Alimentación y Nutrición, 22(1), 29-44.

Bashour, C., Yared, J., Ryan, T., Rady, M., Masha, E., \& Leventhal, M. (2000). Long-term survival and functional capacity in cardiac surgery patients after prolonged intensive care. Critical Care Medicine, 28, 3847-53.

Bristian, B., Blackburn, G., Vitale, J., Cochran, D., \& Naylor, J. (1976). Prevalence of malnutrition in general medical patients. JAMA, 1567-1570.

Christman, J., \& McCain, R. (1993). A sensible approach to the nutritional support of mechanically ventilated critically ill patients. Intensive Care Medicine, 19, 129-136.

Duarte, M., León, D., Larrondo, H., Crespo, A., Segura, R., \& Ernesto, L. (2010). Estado nutricional del paciente sujeto a ventilación mecánica en una unidad de cuidados críticos. Revista Cubana de Alimentación y Nutrición, 20(1), 92-101.

Frutos, F., Alía, A., Valverdu, I., Benito, S., \& Esteban, A. (2003). . Pronóstico de una cohorte de enfermos en ventilación mecánica en 72 unidades de cuidados intensivos en España. Medicina Intensiva, 27(3), 162-8.

Gutierrez, J., Serralde, A., \& Guevara, M. (2007). Prevalencia de desnutrición del adulto mayor al ingreso hospitalario. Nutrición Hospitalaria, 22, 702-709.

Hernández, W., Jiménez, C., Parellada, J., González, I., \& Amador, A. (2011). Diferentes métodos de evaluación nutricional en pacientes graves sometidos a ventilación mecánica invasiva. Revista Cubana de Medicina Intensiva, 10(3), 71-89.

Heyland, D., Konopad, E., Noseworthy, T., Johnston, R., \& Gafni, A. (1998). . Is it worthwhile' to continue treating patients with a prolonged stay ( $>14$ days) in the ICU? An economic evaluation. Chest, 114, 192-8.

Hill, G., Blacket, A., Pickford, I., Burkinsshaw, L., Young, C., \& Schorah, C. (1977). Malnutrition in surgical patients. An unrecognised problem. The Lancet, 26, 689-692.

Hughes, M., MacKirdy, F., Norrie, J., \& Grant, I. (2001). Outcome of long-stay intensive care patients. Intensive Care Medicine, 27, 779-82.

Kelly, I., Tessier, S., \& Cahill, A. (2000). Still hungry in hospital: identifying malnutrition in acute hospital admissions. QJ Med, 93, 93 - 98.

Marik, P., \& Flemmer, M. (2012). Immunonutrition in the surgical patient. Minerva Anestesiol, 78, 336-42.

Mault, J. (2000). Energy balance and outcome in critically ill patients: results of a multicenter, prospective, randomized trial by the ICU Nutrition Study Group. Journal Parenteral Nutrition, 24, S24.

Montuclard, L., Garrouste-Orgeas, M., Timsit, J., Misset, B., De jonghe, B., \& Carlet, J. (2000). Outcome, functional autonomy, and quality of life of elderly patients with a long-term intensive care unit stay. Critical Care Medicine, 28, 3389-95.
Naber, T., Schermer, T., de Bree, A., Nusteling, K., Eggink, L., Krumei, J., y otros. (1997). Prevalence of malnutrition in nonsurgical hospitalizaed patients and its association with disease complications. Am J Clin Nutri, 66(5), 1232-9.

Norman, K., Pichard, C., Lochs, H., \& Pirlich, M. (2008). Prognostic impact of disease-related malnutrition. Clinical Nutrition, 27, 5-15.

Ortiz-Saavedra, P., Manrique-Hurtado, H., Solis-Villanueva, J., Candiotti-Herrera, M., Ige-Afuso, M., \& Torres-Ruiz, C. (2007). Prevalencia de desnutrición en los servicios de hospitalización de medicina. Rev Soc Per Med Interna, $20,17-21$

Pingleton, S. (1990). Enteral nutrition and infection in the intensive care unit. Semin Resp Infect, 5, 185-190.

Rogers, R., Donahoue, M., \& Constantino, J. (1992). Physiologic effects of oral supplemental feeding in malnourished patients with chronic obstructive pulmonary disease. A randomized Control study. Am Rev Respir Dis, 146, 1511-1517.

Ryan, C., Road, J., Buckey, P., Ross, C., \& Whittaker, J. (1993). Energy balance in stable malnourished patients with chronic obstructive pulmonary disease. Chest, 103(1), 1038-1044.

Santana-Cabrera, G., Shanahan, O., García, M., Ramirez, A., Sánchez, M., \& Hernández, E. (2006). Calidad del soporte nutricional artificial en una unidad de cuidados intensivos. Nutrición Hospitalaria, 21(6), 661-666.

Sullivan, D., Sun, S., \& Paredes, R. (1999). Protein-energy undernutrition among elderly hospitalized patients: a prospective study. JAMA, 281(21), 2013-9.

Vásquez, J., Davas, R., \& Reyes, E. (2012). Estado nutricional de pacientes en una unidad de cuidados intensivos. Revista Cubana de Medicina Intensiva, 11(3).

Vidal, A., Iglesias, M., Pertega, S., Ayúcar, A., \& Vidal, O. (2008). Prevalencia de malnutrición en los servicios médicos y quirúrgicos de un hospital universitario. Nutrición Hospitalaria, 23(3).

Villalobos, J., García-Almeida, J., Guzmán, J., Rioja, R., Osorio, D., Rodríguez-García, L., y otros. (2006). Proceso INFORNUT ${ }^{\circledR}$ : validación de la fase de filtro -FILNUT - y comparación con otros métodos de detección precoz de desnutrición hospitalaria. Nutricion Hospitalaria, 21, 491-504.

Villet, S., Chiolero, R., Bollmann, M., Revelly, J., Cayeux, R., Delaure, J., y otros. (2005). Negative impact of hypocaloric feeding and energy balance on clinical outcome in ICU patients. Clin Nutr, 24, 502-9.

Waitzberg, D., Caiaffa, W., \& Correia, I. (2001). Hospital Malnutrition: The Brazilian National Survey (IBRANUTRI): A study of 4000 patients. Nutrition, 17, 573 - 580 .

Wyszynski, D., Perman, M., \& Crivelli, A. (2003). Prevalence of Hospital Malnutrition in Argentina: Preliminary Results of a Population-Based Study. Nutrition, 19, 115 - 119.

Recibido: 23/01/2015

Aceptado: 25/05/2015 